\title{
¿INFLUYE EL AFECTO SOBRE LA ESTIMACIÓN DEL TIEMPO? UN ESTUDIO EXPERIMENTAL
}

\author{
Verónica Cervigón-Carrasco \\ SALUSEX-Dpto. de Psicología Básica, Clínica y Psicobiología \\ Universitat Jaume I, Spain \\ Jesús Castro-Calvo \\ SALUSEX-Dpto. de Personalidad, Evaluación y Tratamientos Psicológicos \\ Universitat de València, Spain \\ Rafael Ballester-Arnal \\ SALUSEX-Dpto. de Psicología Básica, Clínica y Psicobiología \\ Universitat Jaume I, Spain \\ Marta García-Barba \\ SALUSEX-Dpto. de Psicología Básica, Clínica y Psicobiología \\ Universitat Jaume I, Spain \\ María López-Fando Galdón \\ SALUSEX-Dpto. de Psicología Básica, Clínica y Psicobiología \\ Universitat Jaume I, Spain
}

Recepción Artículo: 6 febrero 2020

Admisión Evaluación: 4 marzo 2020

Informe Evaluador 1: 1 enero 2020

Informe Evaluador 2: 1 enero 2020

Aprobación Publicación: 20 abril 2020

\section{RESUMEN}

Introducción: Las alteraciones en la percepción del paso del tiempo y en la estimación de su duración han sido ampliamente estudiadas, especialmente en patologías donde la afectividad es protagonista. Se sugiere que, en trastornos marcados por el afecto negativo, se tendería a sobreestimar el tiempo y percibir que pasa más lento, ocurriendo lo contrario en trastornos marcados por el afecto positivo. Sin embargo, estudios recientes presentan hallazgos contradictorios, sugiriendo que el afecto o no es tan significativo o depende de otras condiciones (p.e., exposición a contenido estimular concurrente). El objetivo de este trabajo es analizar si el afecto influye sobre la precisión en la estimación de la duración del tiempo. Método: 97 participantes (56,7\% mujeres) entre 18 y 65 años cumplimentaron el PANAS y se sometieron a una tarea experimental. Ésta consistía en la exposición a diferentes contenidos (series de TV, videojuegos y documentales) en 4 condiciones temporales (60, 90, 120 y 150 segundos). Tras cada exposición, los participantes estimaban su duración (en segundos) y valoraban cómo de rápido se les había pasado el tiempo. Resultados: Mientras que la afectividad negativa no influía sobre la estimación de la duración o el paso del tiempo, los participantes con una elevada afectividad positiva percibían que el tiempo pasaba más lento cuando eran expuestos a series de TV $(t=2,22 ; p=, 043)$. Estos participantes tendían también infravalorar la duración de cada video (especialmente, las series), si bien esta tendencia no era significativa ( $r=-$ $0,188 ; p=0,065)$. Conclusiones: Si bien identificamos una cierta influencia del afecto positivo sobre la percepción 


\section{¿INFLUYE EL AFECTO SOBRE LA ESTIMACIÓN DEL TIEMPO? UN ESTUDIO EXPERIMENTAL}

de la duración y el paso del tiempo, este estudio refutaría que el afecto medie significativamente sobre estos aspectos. Esto apoyaría la necesidad de revisar las teorías que defienden una relación táctica entre afecto y alteraciones en la percepción y el paso del tiempo.

Palabras clave: duración del tiempo; paso del tiempo; afecto positivo; afecto negativo

\section{ABSTRACT}

Does affectivity influence on time estimation? an experimental study. Introduction: Disruptions in passage time percepction and its duration estimation had been widely studied, particularly in pathologies where affectivivity plays the lead. It is suggested that mental illnesses characterised by negative affect are related to overestimating of time duration and slower down passage time, while in mental ilnesses characterized by positive affect the opposite is usually the case. Nevertheless, recent research come out with contradictory findings, suggesting that affect is not significant or it depends of another conditions (p.e. exposition to concurrent stimular content). The main purpose of this paper is to analyze if affect has effect on time estimation and the passage time perception. Method: 97 participants (56,7\% women), whose age oscillates between 18 and 65 years old completed PANAS questionnaire and they were the subject of an experimental task. The experimental task consisting in four sets, where different contents (sitcoms, videogames and dcomentary films) were exposed to participants in four timing conditions (videos of 60, 90,120 and 150 seconds). Following to each exposition, participants tried to estimate the video duration (in seconds) and they valued how fast was the passage time for them. Results: While negative affectivity had not influence on time estimation or percepción of the time passage, participants who report high positive affect present a lower passage time perception in exposition to TV sitcoms $(t=2,22 ; p=0.043)$. These participants tended to underestimate the duration of each video (especially, sitcoms), despite the fact that this shift was not significative $(r=-0,188 ; p=0,065)$. Conclusions: Although we have detected some influence of postiive affect on duration's perception and time passage, this study disputes that affect has some significant paper on these aspects. This finding supports the need of review theories that defens a tactical relationship between affect and disruptions in time estimation and perception.

Keywords: time duration; time passage positive affect; negative affect

\section{INTRODUCCIÓN}

Las alteraciones en los dos procesos básicos de percepción del tiempo (es decir, la estimación de su duración y la percepción subjetiva de su paso) han sido ampliamente estudiadas, especialmente en el contexto de las enfermedades mentales graves (Oyánadel \& Buela-Casal, 2014). Existe un cierto consenso en la literatura a la hora de afirmar que estos procesos y su correcto funcionamiento tienen un valor claramente adaptativo, puesto que juegan un papel fundamental en el control de su conducta y su adecuación al ambiente que le rodea (EspinosaFernández \& Buela-Casal, 2002). Un ejemplo de su relevancia sería su activación a la hora de realizar cálculos y estimaciones temporales, que permitan por ejemplo, llegar puntual a nuestro puesto de trabajo. Se sugiere que el mecanismo que subyacería a la capacidad de control temporal residiría en la existencia de un reloj interno cuya tarea es producir y codificar la información temporal para su posterior cálculo o estimación (Alústiza, et al., 2015; Droit-Volet, Fayolle \& Gil, 2011). En concreto, se sugiere que este reloj podría verse alterado por factores internos (expectativas, rasgos psicopatológicos, personalidad o el estado emocional -afecto-) y factores externos (por ejemplo, la exposición a diferente tipo de contenido estimular) (teoría de la segmentación, Rammsayer \& Brandler, 2007; Poynter, 1983).

En la literatura científica, existen evidencias que avalan que los procesos de percepción temporal se encuentran alterados en un porcentaje importante de los pacientes con trastorno mental grave (Bolbecker et al., 2011; Mahlberg et al., 2008). En concreto, algunos estudios reportan una alteración en la estimación de la duración y en la percepción del paso del tiempo en trastornos como la esquizofrenia y otros trastornos psicóticos, el trastorno bipolar (sobre todo, durante episodios maniáticos), la depresión mayor o los trastornos de personalidad del cluster $\mathrm{B}$, correlacionando incluso con una mayor gravedad de la sintomatología y el deterioro cognitivo (Alústiza, 
et. al, 2015; Bolbecker et al., 2014; Gil \& Droit-Volet, 2009; Kong, 2019; Oyanadel \& Buela-Casal, 2014; Penney et al., 2005).

Esta literatura sostiene que, en general, Ios pacientes con un trastorno mental grave serían menos precisos a la hora de estimar el tiempo y que la falta de precisión se dirigiría a una dirección o a otra (es decir, a la sobreestimación o la infravaloración de la duración del tiempo) en función del trastorno concreto. En cuanto al trastorno depresivo mayor (donde predomina el afecto negativo), los hallazgos apuntan a que se produciría una sobreestimación temporal y una percepción subjetiva del paso del tiempo más lenta (Gil \& Droit-Volet, 2009). Asimismo, se advierte que, a mayor gravedad de la sintomatología, mayor afectación se da sobre la estimación de la duración del tiempo y su paso (Bschor, 2004). Mientras, en trastornos como la esquizofrenia y trastornos afines o en el trastorno bipolar (fase maníaca), las evidencias apuntan mayoritariamente a que sucede un patrón inverso al anterior, lo que quiere decir que estos trastornos se vinculan con una infravaloración temporal, y una percepción subjetiva del paso del tiempo más rápida (Alústiza, 2015; Mahlberg et al., 2008), además de con una mayor variabilidad en las estimaciones (Bolbecker, 2011).

Los estudios que buscan el denominador común que explica su mayor vulnerabilidad a la hora de presentar estas alteraciones temporales, postulan que uno de los posibles factores sería la afectividad (Angrilli et. al, 1997; Penney et al., 2005). Más allá de los trastornos antes citados, la sobreestimación temporal y la percepción subjetiva de lentitud en el paso se ha relacionado con unos mayores niveles de afectividad negativa dentro de rangos más normativos, mientras que la infravaloración temporal y la percepción subjetiva de rapidez de su paso se ha relacionado con los estados afectivos positivos (Droit-Volet, Fayolle \& Gil, 2011; Griffin \& Buehler, 2005; Kong, 2019). Este fenómeno se justificaría aludiendo a los cambios de ritmo que se da en personas afectadas por estos estados afectivos. La afectividad negativa suele ir acompañada por un enlentecimiento generalizado que se extrapolaría al funcionamiento más lento del reloj interno, contribuyendo, por tanto, a una percepción temporal más lenta y a una sobreestimación de la duración del tiempo. La afectividad positiva va acompañada de un patrón rítmico inverso, generándose, por tanto, una aceleración del reloj interno que daría lugar a una mayor rapidez cognitiva y perceptiva que se derivaría en una infravaloración de la duración del tiempo y a que se perciba como más rápido (Alústiza, 2015; Oyánadel \& Buela-Casal, 2014; Tipples, 2008).

A pesar del gran grueso de literatura que defiende y sustenta esta postura, los datos no son concluyentes. Así, existe una serie de estudios que han llegado a resultados contrarios (o no significativos). Este sería el caso de estudios como el de Bschor (2004) o Tushir et al. (2016) en el que se observó que los pacientes con depresión infravaloraban el tiempo. Por otro lado, en los estudios de Sévigny, Everret \& Gordon (2003) y Oberfeld, et al. (2014) no se registraron diferencias en la estimación temporal entre personas con trastorno depresivo y controles (personas sanas). Por tanto, todavía se desconocería el grado en que la afectividad contribuye, si es que realmente lo hace, en el desarrollo de estas alteraciones en la percepción y estimación temporal. Asimismo, hasta el momento

apenas se han realizado estudios que exploren cómo el contexto estimular (es decir, la presencia o ausencia de estímulos en su contexto) influye sobre esta estimación.

En este contexto, el objetivo del presente estudio era explorar el impacto de la afectividad sobre la precisión en la estimación del tiempo y en la percepción subjetiva de la velocidad de su paso. Este análisis se realizó en diferentes condiciones experimentales (es decir, exponiendo a los participantes a diferente contenido estimular), a fin de determinar si hay factores externos que pueden modular estos procesos. A priori, y en base a lo encontrado en los estudios anteriores, se esperaba que el afecto influyera sobre la percepción del tiempo. Concretamente, se esperaba que el afecto negativo se relacionara con una percepción del paso del tiempo más lenta y con una sobreestimación de la duración del tiempo, mientras que el afecto positivo propiciara el efecto contrario. 


\section{¿INFLUYE EL AFECTO SOBRE LA ESTIMACIÓN DEL TIEMPO? UN ESTUDIO EXPERIMENTAL}

\section{MÉTODO}

\section{Participantes}

En este estudio participaron un total de 97 personas, de las cuales 55 eran mujeres (56,7\%) y 42 hombres (43'3\%). Sus edades oscilaban entre los 18 y 65 años, con una edad media de 25,2 años(DT=6,84). En cuanto a su nivel de estudios, observamos que la mayoría de los participantes (63,9\%) poseen estudios de grado/ diplomatura o licenciatura, mientras que un 15,5\% de la muestra se encuentra en posesión de estudios secundarios y un $13,4 \%$ poseen estudios de máster 0 doctorado. Tan solo un $1 \%$ posee estudios primarios y otro $1 \%$ posee estudios de Formación Profesional. Finalmente, el 74,5\% de la muestra se encontraba estudiando, el 18,6\% trabajaba, mientras que tan solo un 5,2\% estaba desempleado y tan solo un 1\% se encontraba en situación de inactividad (jubilación, discapacidad...).

\section{Procedimiento y paradigma experimental:}

Los participantes en este estudio fueron reclutados a través de contacto directo e indirecto (método de bola de nieve); así como mediante apps de mensajería instantánea (Whatssap y Line) y anuncios en diversas redes sociales (Instagram y Facebook). A la hora de seleccionar la muestra, se utilizó como criterio de inclusión el tener una edad superior a 18 años. Tras su reclutamiento, fueron citados, entre finales del año 2018 e inicios del año 2020, de forma presencial e individual en el laboratorio de investigación de la Unidad de Investigación sobre Sexualidad y Sida (Salusex) de la Universitat Jaume I de Castelló. El laboratorio carecía de cualquier objeto que pudiera servir de referencia temporal, a fin de evitar la influencia en sus respuestas durante el experimento (nótese que la tarea experimental se basaba en la estimación del tiempo). Previamente al inicio de la condición experimental y con el fin de resolver posibles dudas, se les ofrecía a los participantes una breve explicación de todo el proceso. Tras esto, se quedaban solos en el laboratorio durante la duración total del experimento para así preservar su intimidad y privacidad.

Todo el paradigma experimental se realizaba asistido por ordenador, minimizando así la intervención del experimentador.

La tarea experimental se componía de 16 ensayos repartidos en 4 series. Antes de empezar la tarea, se presentó una condición control compuesta por una pantalla con un punto negro en el centro; a cada participante se le pedía que presionara un botón cuando pensara que había transcurrido 1 minuto desde la aparición del punto negro. El objetivo era disponer de un indicador objetivo de la precisión en la estimación temporal sin contenido estimular concurrente. En cada una de las 4 series experimentales, se presentaba un vídeo de cada contenido estimular testado (pornografía, series de TV, videojuegos y documentales); la duración podía ser de 60, 90, 120 y 150 segundos, contrabalanceándose la duración de cada contenido en las diferentes tandas. Así, una vez terminada la tarea, cada participante habría sido expuesto a un vídeo del mismo contenido estimular en las cuatro condiciones temporales -(p.e.un vídeo de 60 segundos con una serie de TV, otro de 90, otro de 120 y uno de 150); y lo mismo para cada condición estimular. Después de cada ensayo, los participantes debían: (a) hacer una estimación de la duración del vídeo (en segundos), (b) decir cómo de rápido se les había pasado el tiempo -en una escala Likert de 0 a 10 ( $0=$ muy lento; $10=$ muy rápido)- y (c) valorar el grado de satisfacción con el contenido, también en una escala Likert de 0 a 10 ( $0=$ no me ha gustado nada; 10= me ha gustado mucho). La finalidad de esta tarea era obtener información relativa a la precisión en la estimación de la duración del y la percepción de su duración, para así posteriormente evaluar si había diferencias que se podían explicar por el estado afectivo de la persona (también evaluado) y por el tipo de contenido estimular presentado.

Además, se administró a los participantes una batería de cuestionarios entre los que se encontraba la Positive and Negative Affect Scale (PANAS, Watson, Clark \& Tellegen, 1998; Adaptación española por López-Gómez, Hervás \& Vázquez, 2015), en la que se presentaban un listado de emociones que hacían referencia a las dos dimensiones de la afectividad: afecto positivo y afecto negativo. En cuanto a la fiabilidad de este cuestionario, encontramos que es de ,73 para el afecto positivo y de ,83 para el afecto negativo. Los participantes puntuaban 
del 0 al 4, el grado en el que cada emoción los identificaba en ese momento, y así se evaluaba el nivel de cada dimensión en cada uno. Una particularidad de esta escala es que ambas dimensiones resultan independientes: es decir, que el afecto positivo y en negativo no serían considerados extremos opuestos de un mismo contino, sino que se obtienen puntuaciones independientes para cada escala.

\section{Análisis de datos}

Con el objetivo de explorar si el afecto (tanto el positivo como el negativo) modulaba los procesos de estimación del tiempo y la percepción de su paso, se procedió al cálculo de una prueba t para muestras independientes. A fin de realizar dicho análisis, se dicotomizó la muestra en función de sus puntuaciones en las escalas de afecto positivo y de afecto negativo: Ios que puntuaban por encima del percentil 85 en cualquiera de las dos escalas eran considerados como participantes de un alto afecto (o positivo o negativo), mientras que en la otra categoría se incluía a las personas con un afecto normativo. Estas pruebas se calcularon para todos los contenidos estimulares.

Posteriormente, se llevaron a cabo correlaciones tipo Pearson para determinar la posible relación existente entre el afecto positivo, el negativo y la precisión en la estimación de la duración del tiempo y la percepción de su paso. Esta aproximación permitía un análisis lineal de la influencia entre las variables objeto de estudio (en lugar de una aproximación diferencial como en el caso anterior) Nuevamente, estas pruebas se calcularon para todos los contenidos estimulares.

\section{RESULTADOS}

En la Tabla 1 se presentan los resultados en función de la clasificación percentil de los participantes. Respecto al afecto negativo, no se encuentran diferencias estadísticamente significativas en la precisión en la estimación del tiempo y en la percepción de su paso en ninguna de las condiciones: es decir, que ambos aspectos no diferían en función de si se presentaba un alto afecto negativo. Para el afecto positivo, solo se observan diferencias estadísticamente significativas durante el visionado de series de televisión: en concreto, las personas con una afectividad positiva normativa percibían que el tiempo pasaba más lento durante el visionado de $\operatorname{series}(t=0,22 ; p=0,045)$. 


\section{¿INFLUYE EL AFECTO SOBRE LA ESTIMACIÓN DEL TIEMPO? UN ESTUDIO EXPERIMENTAL}

Tabla 1. Diferencias en la percepción del paso del tiempo entre personas con alto afecto positivo/negativo y el resto de participantes

\begin{tabular}{|c|c|c|c|c|c|c|}
\hline \multicolumn{4}{|c|}{ Afecto positivo } & \multicolumn{3}{|c|}{$\begin{array}{r}\text { Afecto } \\
\text { negativo }\end{array}$} \\
\hline & $\begin{array}{l}M \text { Alto } \\
\text { afecto } \\
\text { positivo } \\
\text { (percentil } \\
>85 \text { ) } \\
\text { (DT } \\
\text { ) }\end{array}$ & $\begin{array}{c}M \text { Resto } \\
\text { de la } \\
\text { muestra } \\
\text { (percentil } \\
<85 \text { ) } \\
\text { (DT } \\
\text { ) }\end{array}$ & Prueba $\mathrm{t}(\mathrm{p})$ & $\begin{array}{l}M \text { Alto } \\
\text { afecto } \\
\text { negativo } \\
\text { (percentil } \\
>85 \text { ) } \\
\text { (DT } \\
\text { ) }\end{array}$ & $\begin{array}{c}M \text { Resto } \\
\text { de la } \\
\text { muestra } \\
\text { (percentil } \\
<85 \text { ) } \\
\text { (DT) }\end{array}$ & $\begin{array}{l}\text { Prueba t } \\
\text { (p) }\end{array}$ \\
\hline \multicolumn{7}{|l|}{$\begin{array}{l}\text { Estimación del } \\
\text { tiempo }\end{array}$} \\
\hline $\begin{array}{l}\text { Condición } \\
\text { control } \\
\text { (círculo } \\
\text { negro) }\end{array}$ & $\begin{array}{r}-3,86 \\
(19,41)\end{array}$ & $4,93(12,36)$ & $\begin{array}{l}t=0,267 \\
(p=0,792)\end{array}$ & $\begin{array}{r}-6,30 \\
(12,46)\end{array}$ & $\begin{array}{r}-3,65 \\
(19,39)\end{array}$ & $\begin{aligned} \mathrm{t} & =0,654(\mathrm{p} \\
& =0,520)\end{aligned}$ \\
\hline $\begin{array}{l}\text { Condición } \\
\text { serie de TV }\end{array}$ & $3,77(37,69)$ & $\begin{array}{r}3,87 \\
(46,65)\end{array}$ & $\begin{array}{l}\mathrm{t}=0,090 \\
(\mathrm{p}=0,993)\end{array}$ & $\begin{array}{r}-1,79 \\
(32,38)\end{array}$ & $4,73(47,16)$ & $\begin{aligned} \mathrm{t} & =0,630(\mathrm{p} \\
& =0,536)\end{aligned}$ \\
\hline Condición & $\begin{array}{r}24,56 \\
58\end{array}$ & 20,25 & $\mathrm{t}=-0,250$ & $\begin{array}{r}12,50 \\
\end{array}$ & $\begin{array}{r}22,12 \\
\end{array}$ & $\mathrm{t}=0,708(\mathrm{p}$ \\
\hline $\begin{array}{l}\text { videojuego } \\
\text { Condición }\end{array}$ & $\begin{array}{r}(58,21) \\
-0,15\end{array}$ & $\begin{array}{r}(49,01) \\
8,58\end{array}$ & $\begin{array}{l}(p=0,804) \\
t=0,702\end{array}$ & $\begin{array}{r}(44,70) \\
10,42\end{array}$ & $\begin{array}{c}(50,92) \\
6,97(4128)\end{array}$ & $\begin{array}{c}=0,489) \\
t=-\end{array}$ \\
\hline documental & $(41,82)$ & $(41,19)$ & $(\mathrm{p}=0,493)$ & $(42,00)$ & & $\begin{array}{c}0,266(\mathrm{p}= \\
0,794)\end{array}$ \\
\hline \multicolumn{7}{|l|}{$\begin{array}{l}\text { Percepción del } \\
\text { paso del } \\
\text { tiempo }\end{array}$} \\
\hline $\begin{array}{l}\text { Condición } \\
\text { serie de TV }\end{array}$ & $5,90(1,97)$ & $7,16(1,45)$ & $\begin{array}{l}t=2,22 \\
(p=0,043)\end{array}$ & $4,17(1,07)$ & $6,97(1,65)$ & $\begin{array}{c}t=- \\
0,575(\mathrm{p}= \\
0,571)\end{array}$ \\
\hline $\begin{array}{r}\text { Condición } \\
\text { videojuego }\end{array}$ & $4,13(1,39)$ & $4,34(1,44)$ & $\begin{array}{l}t=0,48 \\
(p=0,631)\end{array}$ & $4,02(1,45)$ & $4,35(1,43)$ & $\begin{array}{c}\mathrm{t}=0,782 \\
(\mathrm{p}=0,446)\end{array}$ \\
\hline $\begin{array}{r}\text { Condición } \\
\text { documental }\end{array}$ & $4,73(1,37)$ & $4,47(1,32)$ & $\begin{array}{l}t=0,64 \\
(p=0,530)\end{array}$ & $4,29(1,16)$ & $4,54(1,36)$ & $\begin{aligned} \mathrm{t} & =0,706(\mathrm{p} \\
& =0,490)\end{aligned}$ \\
\hline
\end{tabular}

En cuanto a los análisis de correlación (Tabla 2), lo que se encontró fue que el afecto negativo no correlacionaba significativamente ni con la precisión en la estimación del tiempo ni con la percepción de su paso en ninguna de las condiciones experimentales. En cuanto al afecto positivo, tampoco se encuentran resultados significativos, pero sí se observa una tendencia a infravalorar la duración de las series de TV cuando el nivel de afectividad es elevado $(r=-0,188 ; p=0,065)$. 
Tabla 2: Correlaciones entre estimación del tiempo y percepción de su paso y afectividad

\begin{tabular}{|c|c|c|c|c|}
\hline & \multicolumn{2}{|c|}{$\begin{array}{l}\text { Afecto } \\
\text { positivo }\end{array}$} & \multicolumn{2}{|c|}{ Afecto negativo } \\
\hline & $\mathrm{r}$ & $\mathrm{p}$ & $\mathrm{r}$ & $\mathrm{p}$ \\
\hline \multicolumn{5}{|l|}{ Estimación del tiempo } \\
\hline $\begin{array}{l}\text { Condición control } \\
\text { (círculo } \\
\text { negro) }\end{array}$ & $-0,041$ & $\begin{array}{c}0,68 \\
8\end{array}$ & $-0,004$ & 0,971 \\
\hline Condición serie de TV & $-0,106$ & $\begin{array}{c}0,30 \\
2\end{array}$ & 0,156 & 0,127 \\
\hline Condición videojuego & 0,058 & $\begin{array}{c}0,57 \\
3\end{array}$ & 0,146 & 0,154 \\
\hline Condición documental & 0,039 & $\begin{array}{c}0,70 \\
8\end{array}$ & 0,073 & 0,481 \\
\hline \multicolumn{5}{|l|}{$\begin{array}{l}\text { Percepción del paso del } \\
\text { tiempo }\end{array}$} \\
\hline Condición serie de TV & $-0,188$ & $\begin{array}{c}0,06 \\
5\end{array}$ & $-0,033$ & 0,745 \\
\hline Condición videojuego & $-0,066$ & $\begin{array}{c}0,52 \\
1\end{array}$ & $-0,088$ & 0,389 \\
\hline Condición documental & $-0,056$ & $\begin{array}{c}0,58 \\
6\end{array}$ & $-0,048$ & 0,638 \\
\hline
\end{tabular}

\section{CONCLUSIONES}

Este estudio pretende arrojar luz sobre el papel del afecto en la estimación de la duración del tiempo y en la percepción de su paso. Este tema ha generado un notable interés, con posturas y resultados encontrados. A tal fin, hemos explorado la precisión en la estimación del tiempo y en la percepción subjetiva de su paso en una muestra de 97 participantes. Este análisis se ha llevado a cabo mediante la implementación de unatarea experimental compuesta por materiales de diferente contenido estimular y duración, y se ha evaluado el nivel de afecto positivo y negativo en cada participante. A la luz de los datos obtenidos, podemos decir que la hipótesis de partida (es decir, que el afecto influye sobre la estimación de la duración del tiempo y la percepción de su paso) no se cumple.

Por tanto, la principal conclusión que podemos extrapolar de este estudio es que el afecto no es un predictor eficaz de la falta de precisión en la estimación temporal. Esto, nos situaría en la misma postura que los estudios con pacientes con trastorno depresivo mayor de Sévigny, Everret \& Gordon (2003) o Bschor (2004) anteriormente citados. Incluso nuestros datos contradirían lo encontrado por Bschor (2004): mientras que este observó que los pacientes depresivos (es decir, con una marcada afectividad negativa) presentaban una infravaloración temporal, nuestros resultados no encuentran relaciones significativas en ninguna dirección ni tampoco diferencias en las estimaciones entre los pacientes con una elevada afectividad negativa y el resto. En este sentido, estos resultados serían similares a los que encontraba Sévigny (2003) en su estudio, confirmando que el afecto negativo no influye en la precisión temporal ni en su alteración.

Por lo que refiere a la afectividad positiva, a pesar de que no encontramos datos robustos ni significativos, sí que hallamos una tendencia a que aquellas personas que presentaban un estado afectivo elevado en el momento de la evaluación, percibían que la duración de la condición series de TV era menor de lo que en realidad era. Sin embargo, y paradójicamente, reportaban una percepción subjetiva de menor velocidad del paso del tiempo, es 


\section{¿INFLUYE EL AFECTO SOBRE LA ESTIMACIÓN DEL TIEMPO? UN ESTUDIO EXPERIMENTAL}

decir, para ellos el tiempo pasaba más lentamente ante el visionado de series de TV. Este hallazgo significaría que el efecto de la afectividad sobre la temporalidad se podría ver mediatizado por el tipo de contenido estimular concurrente.

Una posible explicación sería que las series de TV generalmente evocan emociones positivas. Por tanto, este sería un contenido estimular satisfactorio y concordante con la afectividad positiva. De esta manera y ante la evocación de emociones positivas, se generaría un efecto gratificante que reafirmaría y potenciaría los niveles elevados en afecto positivo ya presentes (Flayelle, et al., 2018). Aquello que justifica o reafirma cómo nos sentimos y, que además, nos resulta agradable y gratificante, acapara y consume una serie de recursos tanto a nivel cognitivo como atencional, impidiendo que estén disponibles para otro tipo de estímulos o tareas como, en este caso, manejar el control del paso del tiempo. Esta desviación de los recursos generaría la pérdida sobre la noción del tiempo (Flayelle, et al., 2018; Orosz, Böthe \& Tóth-Király, 2016; Tóth-Király, et al., 2017). De hecho, en un estudio reciente realizado por Castro et al. (2019) en el que se explora el consumo de series y sus factores asociados, se observa que los participantes experimentaban un aumento de los niveles de afecto positivo derivados por el consumo de series y, además, que hasta un 23,8\% informaba que no había sido completamente consciente del paso del tiempo, frente a solo el $52,5 \%$ de la muestra que aseguraba haberlo sido completamente. Sin duda alguna, un fenómeno que sería interesante abordar con mayor profundidad en futuros estudios es la percepción de mayor lentitud en el paso del tiempo ante las series en presencia de una afectividad positiva elevada, aun presentándose una infravaloración en su duración. Sería conveniente explorar este aspecto, por ir en contra de lo hallado en la literatura científica revisada, y por lo contradictorio que resulta que una percepción del paso del tiempo más lenta vaya aparejada a una infravaloración en su duración.

Una posible limitación de este estudio estaría relacionada con el tamaño de la muestra: aunque 97 participantes es una muestra considerable para un estudio experimental, una muestra de mayor tamaño podría arrojar datos más potentes y robustos que permitirían proponer explicaciones y conclusiones de mayor calado. Además, sería conveniente replicar este estudio con muestra clínica, puesto que en este estudio se ha trabajado la afectividad desde una perspectiva no patológica; es decir, Ios participantes reportaban su estado afectivo más alto 0 más bajo pero dentro de un umbral normativo. Otro motivo que nos llevaría a la replicación de este estudio con muestra clínica es que cabe la posibilidad que los datos obtenidos difieran de los encontrados en este estudio, y que incluso, nos den respuesta a lo encontrado en relación a la incógnita que se ha generado a partir de este estudio asociada a la percepción del paso más lento del tiempo ante las series de TV cuando se reportan niveles más altos de afecto positivo. Este estudio y la implementación de las mejoras comentadas, resulta fundamental para así poner en perspectiva la verdadera asociación entre el afecto y las estimaciones temporales, haciendo quizás necesario reformular las aproximaciones teóricas sobre las que se sustentan los programas de evaluación e tratamiento, con objetivo de realizar intervenciones de mayor eficacia.

\section{REFERENCIAS BIBLIOGRÁFICAS}

Alústiza, I., Pujol, N., Molero, P., \& Ortuño, F. (2015).Temporal processing in schizofrenia: Review. Schizofrenia Reseach: Cognition, X, XXX- XXX

Angrilli, A., Cherubini, P., Pavese, A., \& Manfredini, S. (1997). The influence of affective factors on time perception. Perception \& Psychophysics, 59, 972-982

Bschor, T., Ising, M., Bauer, M., Lewitzka, U., Skerstupeit, M., MüllerOerlingghausen, B., \& Baethge, C. (2004). Time experience and time judgement in major depression, mania and healthy subjects.A controlled study of 93 subjects. Acta Psychiatrica Scandinavica, 109(3), 222-229

Bolbecker, A.R., Westfall, D.R., Howell, J.M., Lackner, R.J., Carroll, C.A. O'Donell, B.F., \& Hetrick, W.P (2014). Increased Timing Variability in Schizofrenia and Bipolar Disorder. Plos ONE 9(5): e97964. doi:10.1371/journal.pone.0097964 
Bolbecker, A.R., Hong, S.L, Kent, J.S., Forshyt, J.K., Klaunig, M.J., Lazar, E., O'Donnell, B.F., \& Hetrick, W.P. (2011). Paced finger-tapping abnormalities in bipolar disorder indicate timing dysfunction. Bipolar Disorder, 13(1), 99-110

Castro, D., Rigby, J., Cabral, D., \& Nisi, V. (2019). The Bing-watcher's journey: Investigating motivations, contexts, and affective states surrounding Netflix viewing. Convergence: The International Journal of Reseach into New Media Technologies, XX(X) 1-18; Doi: 10.1177/1354856519890856

Droite-Volet, S., Fayolle, S.L. \& Gil, S. (2011). Emotion and time perception: Effects of film-induced mood. Frontiers in Integrative Neuroscience, 5, 33

Espinosa-Fernández, L.\& Buela-Casal, G. (2002). La capacidad para percibir y estimar el tiempo en humanos. Suma Psicológica, 9(1), 55-80

Flayelle, M., Canale, N., Vögele, C., Karila, L., Maurage, P., \& Billieux,

J. (2018). Assessing Bing-watching behaviours: Development and Validation of the "Watching TV Series Motives" and "Bing-Watching Engagement and Symptoms" questionnaies. Computers in Human Behaviour, 90, 2636

Gil, S., \& Droit-Volet, S. (2009) Time perception, depression and sadness.

Behavioral Processes, 80(2), 169-176

Griffin, D. \& Buehler, R. (2005). Collaborative planning and prediction: Does group discusión affect optimistic biases in time estimation? Organizational Behavior and Human Decission Processes, 97, 47-63

Kong, R. (2019). The Role of Time Perception in Depression. Journal of Depression adn Anxiety, 8(4), 1-3

López-Gómez, I; Hervás, G. \& Vázquez, C. (2015). Adaptación de la "Escala de afecto positivo y negativo" (PANAS) en una muestra general española. Psicología conductual, 23(3), 529-548

Mahlberg, R., Kienast, T., Bschor, T., Adli, M. (2008). Evaluation of time memory in acutely depressed patients, maniac patients, and healthy controls using a time reproduction task. European Psychiatry, 23(6), 430-433

Oberfeld, D., Thönes, S., Palayoor, D.J., \& Hecht, H. (2014). Depression does not affect time perception and timeto-contact estimation. Frontiers in Psychology, 5, D0I: 3389/fpsyg.2014.00810

Orosz, G., Böthe, B., \& Tóth-Király, I. (2016). The development of Problematic Series Watching Scale (PSWS). Journal of Behavioral Addictions, 5(1), 144-150

Oyanadel, C. \& Buela-Casal, G. (2014). Percepción del tiempo y psicopatología: Influencia de la orientación temporal en la calidad de vida de los trastornos mentales graves. Actas Españolas de Psiquiatría, 42(3), 99-107

Penney, T.B., Meck, W.H., Roberts, S.A., Gibbon, J., \& Erlenmeyer- Kimling, L., (2005). Intervanl-timing deficits in individuals at high risk for schizophrenia. Brain Cognition, 58, 109-118

Poynter, W.D. (1983). Duration judgment and segmentation of experience,

Memory and Cognition. 11, 77-82

Ranmsayer, T.H. \& Brandler, S. (2007). Performance on temporal information processing as a index of general intelligence. Intelligence, 35, 129- 139

Sévigny, M.C., Everett, J., \& Gordon, S. (2003). Depression, attention and time estimation. Brain Cognition, 53(2), $351-353$

Tóth-Kiraly, I., Böthe, B., Tótk-Fáver, E., Hága, G. \& Orosz, G. (2017). Connected to TV series: Quantifying series watching engagement. Journal of Behavioral Addictions, 6(4),472-489

Tipples, J. (2008). Negative emotionality influences the effects of emoción on time perception. Emotion, 2008, 8(1), 127

Tushir, B., Pasipanodya, E.T.T.M., Kymar, Y., \& Singh, T. (2016). Depression and Perception Time. Indian Journal of Social Science Researches, 13(1-2), 7-12

Watson, J.D., Clark, L.A., \& Tellegen, A. (1998). Development and validation of brief mesures of positive and negative affect: The PANAS scales. Journal of Personality and Social Psychology, 54(6), 1063-1070 
